\title{
In vitro Anti-oxidant Potential of New Metabolites from Hypericum oblongifolium (Guttiferae)
}

Naila Raziq, Muhammad Saeed, Muhammad Shaiq Ali, Salman Zafar and Muhammad Imran Ali

\begin{abstract}
Phytochemical investigations on Hypericum oblongifolium led to the isolation of a flavone named folicitin (1) and a bicyclic conjugated lactone, folenolide (2) from the ethyl acetate fraction of methanolic extract. Both metabolites were characterized as new compounds based on detailed spectroscopic analyses. In vitro anti-oxidant potential of both the compounds was evaluated by the DPPH radical scavenging assay. Compound $\mathbf{1}$ exhibited significant antioxidant activity while compound 2 was found inactive.
\end{abstract}


Table S1: ${ }^{1} \mathrm{H}$ and ${ }^{13} \mathrm{C}-\mathrm{NMR}$ data of folecitin (1)

\begin{tabular}{|c|c|c|}
\hline POSITION & ${ }^{1} \mathbf{H}$ & ${ }^{13} \mathrm{C}$ \\
\hline 1 & - & - \\
\hline 2 & - & 158.6 \\
\hline 3 & - & 136.2 \\
\hline 4 & - & 179.7 \\
\hline 5 & - & 163.3 \\
\hline 6 & $6.19(\mathrm{~d}, J=2.0 \mathrm{~Hz})$ & 99.8 \\
\hline 7 & - & 145.6 \\
\hline 8 & $6.37(\mathrm{~d}, J=2.0 \mathrm{~Hz})$ & 94.7 \\
\hline 9 & - & \\
\hline 10 & - & 105.9 \\
\hline 1 ' & - & 122.9 \\
\hline 2 ' & $7.33(\mathrm{~d}, J=2.0 \mathrm{~Hz})$ & 116.9 \\
\hline 3, & - & 145.6 \\
\hline 4 ' & - & 149.8 \\
\hline 5 & $6.90(\mathrm{~d}, J=8.0 \mathrm{~Hz})$ & 116.4 \\
\hline 6 & $7.30(\mathrm{dd}, J=8.0,2.0 \mathrm{~Hz})$ & 122.8 \\
\hline 1, & & - \\
\hline $2 "$ & $5.34(\mathrm{~d}, J=1.5 \mathrm{~Hz})$ & 103.5 \\
\hline $3 "$ & $4.21(\mathrm{dd}, J=3.5,1.5 \mathrm{~Hz})$ & 71.9 \\
\hline $4 "$ & $3.39(\mathrm{~m})$ & 73.2 \\
\hline 5 " & $3.73(\mathrm{dd}, J=9.5,3.5 \mathrm{~Hz})$ & 72.0 \\
\hline $6 "$ & $3.42(\mathrm{~m})$ & 72.1 \\
\hline $7^{\prime \prime}$ & $0.93(\mathrm{~d}, J=6.0 \mathrm{~Hz})$ & 17.7 \\
\hline
\end{tabular}


Table S2: ${ }^{1} \mathrm{H}$ and ${ }^{13} \mathrm{C}$-NMR data of folenolide (2)

\begin{tabular}{|c|c|c|}
\hline POSITION & ${ }^{\mathbf{1}} \mathbf{H}$ & ${ }^{{ }^{3} \mathbf{C}}$ \\
\hline 1 & - & - \\
\hline 2 & $3.98(\mathrm{~m})$ & 69.0 \\
\hline 3 & $3.73(\mathrm{q}, J=4.0 \mathrm{~Hz})$ & 73.6 \\
\hline 4 & $4.40(\mathrm{t}, J=4.5 \mathrm{~Hz})$ & 68.4 \\
\hline 5 & $6.75(\mathrm{t}, J=2.0 \mathrm{~Hz})$ & 139.3 \\
\hline 6 & - & 133.0 \\
\hline 7 & - & 173.0 \\
\hline 8 & $2.18(\mathrm{ddt}, J=17.5,6.5,1.5 \mathrm{~Hz}), 2.70(\mathrm{ddt}, J=$ & 33.0 \\
\hline
\end{tabular}




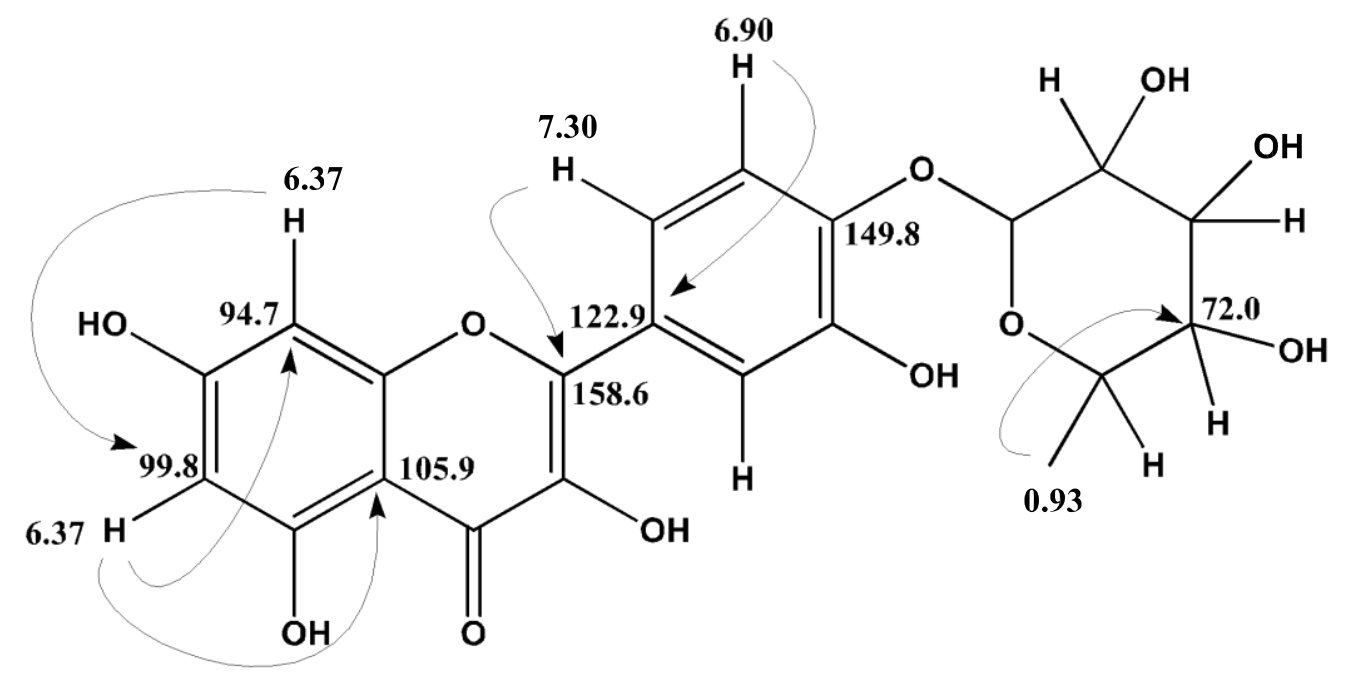

Figure S1: Key HMBC correlations in $\mathbf{1}$

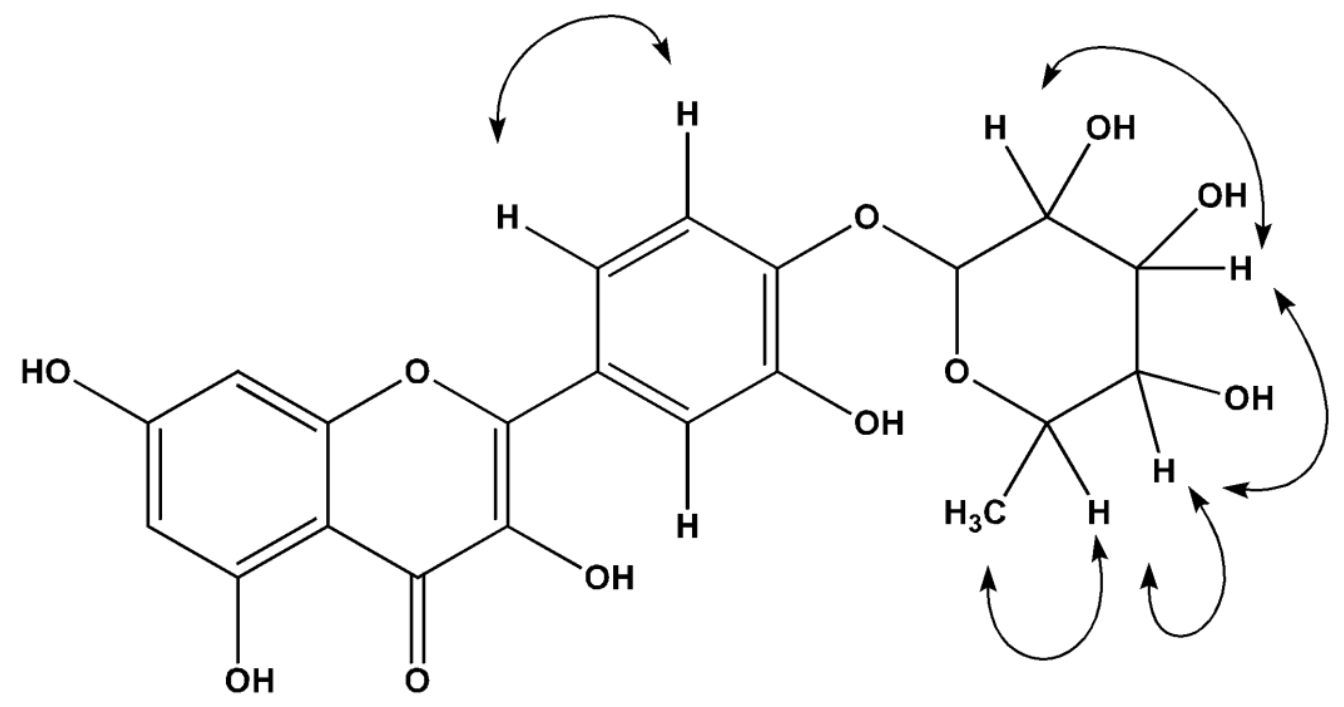

Figure S2: COSY correlations in $\mathbf{1}$ 


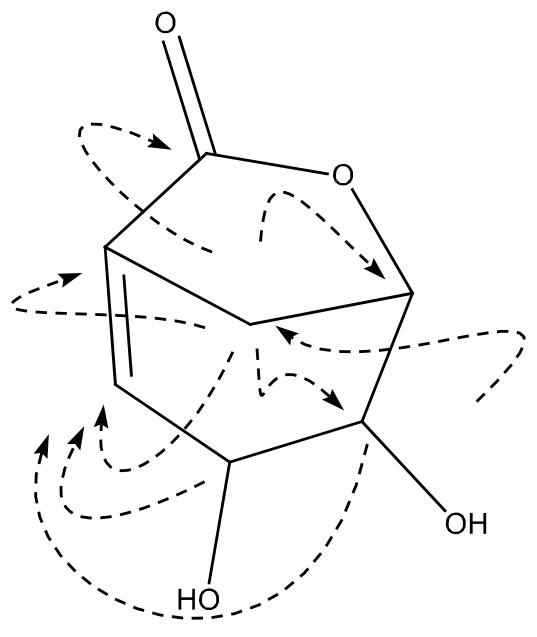

Figure S3: Key HMBC correlations in 2

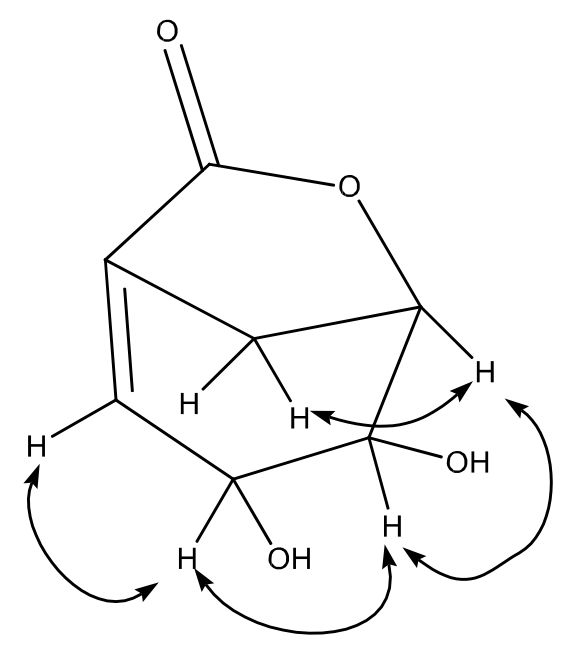

Figure S4: COSY interactions in compound 2 
$1 \mathrm{H}$

स⿴囗十

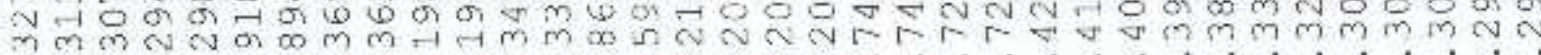

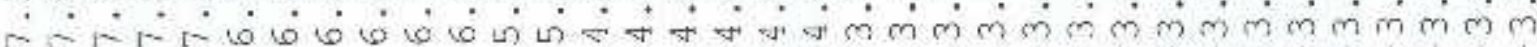

$\{4 \mid$ ।

$m$ in

o. on

$\dot{0} \dot{0}$

NADE

XENO
PROCNO

Date

Time-

INSTRUP

IROBHD

FULEROC

ID

No

NR-03 = Compound 1

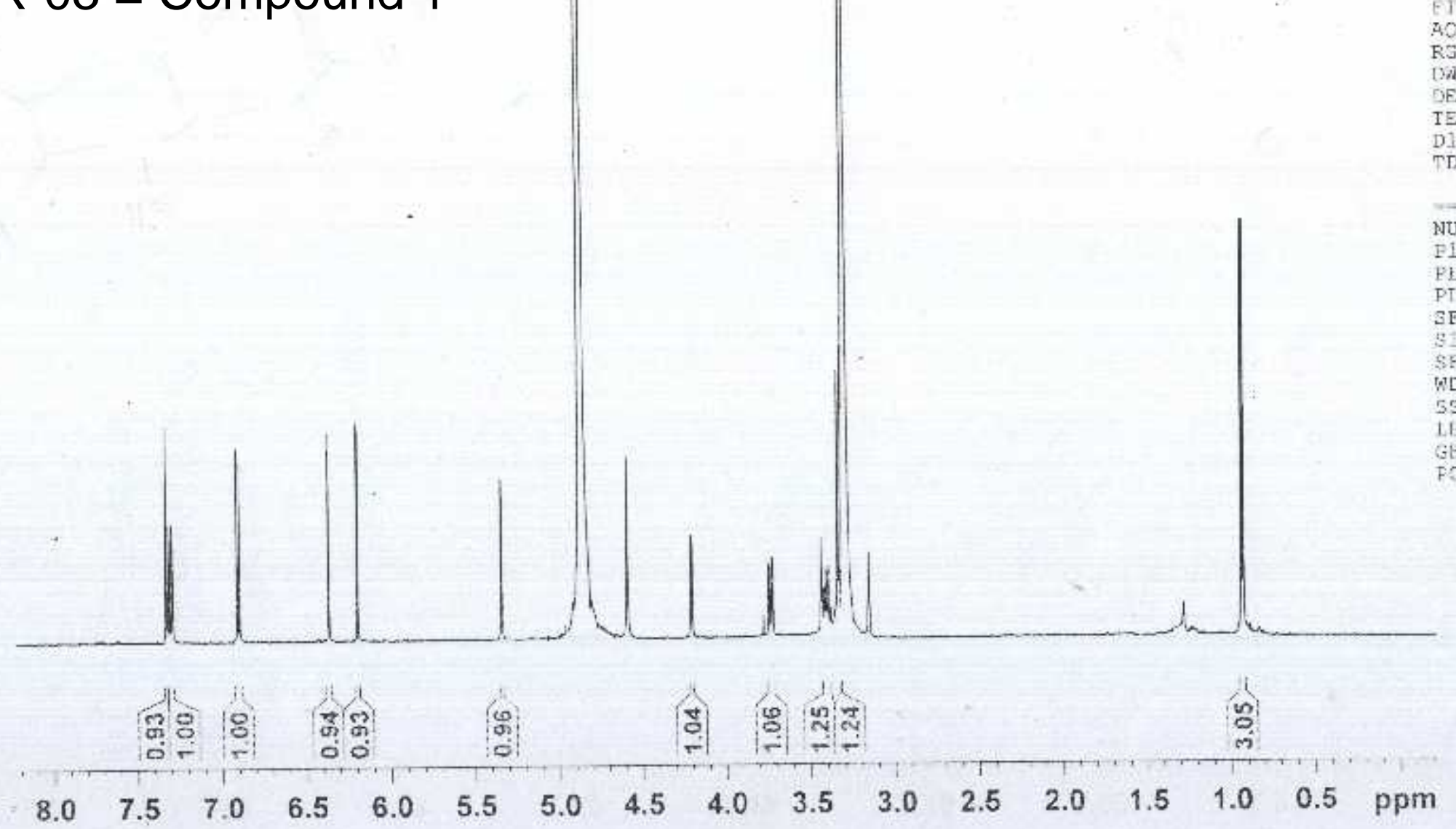


NAILA RAZIQ/DR.SHAIQ/NR-03

$\mathrm{BB}$

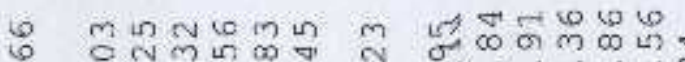

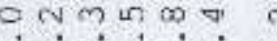

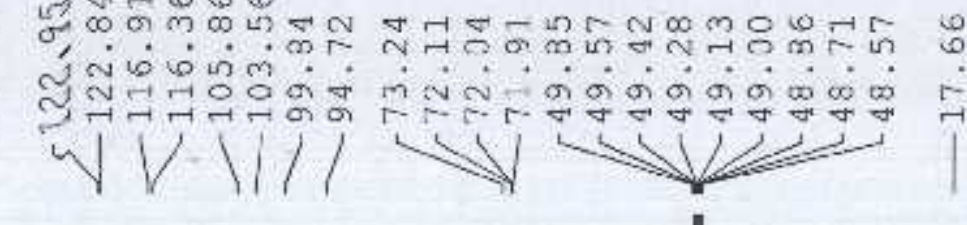

PROCNO

Date

'Irime-

INSTRUY

PROBID
PLLLFCK

PULP

TD

NS

DS

SWT:
ZID RES

$\mathrm{AQ}$

RG

DW

DE

E1

L11

$====$

NบE1

Fi

$1 \pm 1$

GFol

dec:33-13 $20131224^{1}$ 6.29 5. mu CPTCT $2 \mathrm{H}-$

zgpg

$$
\begin{array}{r}
z 909 \\
327.68 \\
\text { Meop } \\
12288
\end{array}
$$

$35971.223 \mathrm{~km}$ $1.097755 \mathrm{kz}$ $0.4555391 \approx e c$ 32708 13.900 usec 6.50 usec 2.00000000 sec $0.03000000 \mathrm{sec}$

\section{$====$}

CPDPRG

NuO2

DCED2

PI?

PLi2

EII 13

PL2W

PLI2W

PIIJW

$\mathrm{P}=136$
$\mathrm{~A}: 02$

SEO
SI
SE

WDV

LB

GB

GC

$=$ CHANREL $f 1$ 12

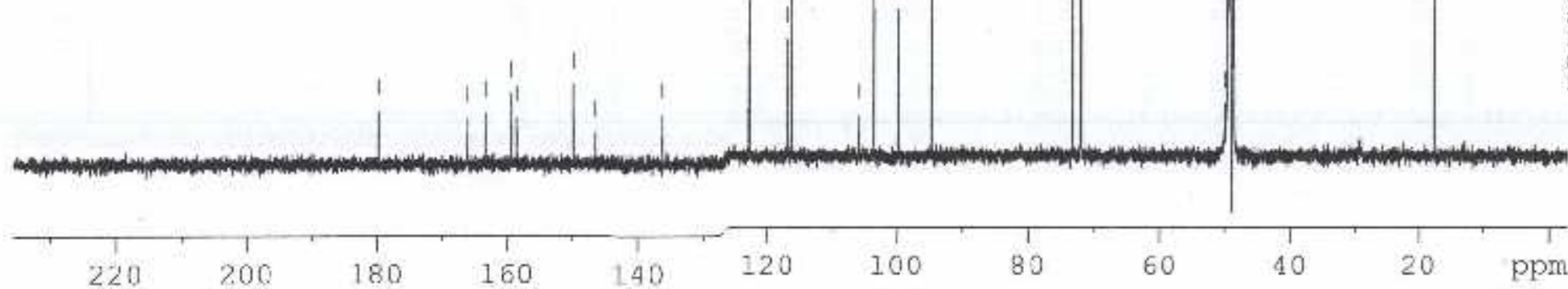




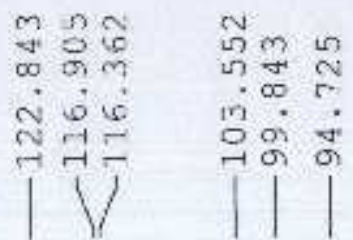

NAILA RAZIO/DR. SHAIQ/NR-03

deptsp135

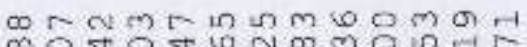

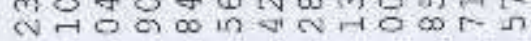
ल w व

5
0
5
5

\section{LAB NOO; 20?}

\begin{tabular}{|c|c|c|c|}
\hline NAME: & & $\operatorname{dec} 23-13$ & \\
\hline ExpNo & & & \\
\hline PROCAC & & 1 & \\
\hline Date & & 20131224 & \\
\hline Time & & $14.5 \%$ & \\
\hline INETBDM & & spect & \\
\hline PROSED & $5 \mathrm{~mm}$ & CPocI Ift- & \\
\hline PIIT.PR.OG & & deptap 135 & \\
\hline $\mathrm{TD}$ & & 32768 & \\
\hline EOLWEUT & & Yeon & \\
\hline NE & & 2534 & \\
\hline DE & & 2 & \\
\hline$\triangle W H$ & & $303 \mathrm{c} 3.231$ & $\mathrm{~Hz}$ \\
\hline FTDRAS & & 0.924775 & 32 \\
\hline$A Q$ & & 0.5407365 & sec \\
\hline $\mathrm{FG}$ & & 32768 & \\
\hline ก\% & & 16.500 & asec \\
\hline$D E$ & & 6.50 & asec \\
\hline$T E$ & & 297,3 & $x$ \\
\hline CNST2 & & 45.0000000 & \\
\hline DI & & 1.50000000 & sec: \\
\hline 02 & & 0.00344928 & $\sec$ \\
\hline $\mathrm{DI2}$ & & 0.00002000 & aec. \\
\hline
\end{tabular}
$\cos$

Noci

21

PL1

PI.

TL1:

$5 F 01$

$\sin 2$

SPNART2

SgOEES2

CHBWNEL $f$ 6

$=====$

GuC2

P4

PCPD2

Th2

L12

PL 2N

SLO2

$\mathrm{SE}$

WDW

s.

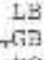

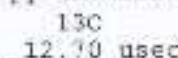

12.10 used 120.00 use $120.003 \mathrm{~B}$

$0.00000000 \pi$ B1.92.15314 $\%$ $-50.99275 \mathrm{~L} \mathrm{MHz}$ 1.29 di

$$
0.500
$$

$0.00 \mathrm{~Hz}$

$=$ CHSRIEL

waItz-E

B. 00 user

8.00 used:

16.00 useC
80.0 1

$3.31 \mathrm{~dB}$

73.31 in

$6.75873323 \pi$

c. 06798734 \%

oro 0230002 रा:

250-8974505 ME:z

EX

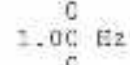

5.40 


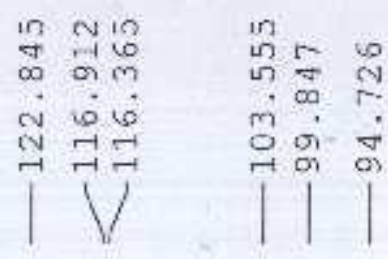

NAILA RAZIQ/DR. SHAIQ/NR-03

deptsp 90

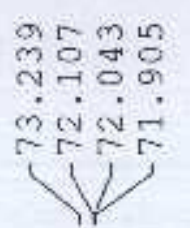

एक m 1089

แल लू

$\sigma \dot{\sigma} \dot{\theta} \dot{0}$

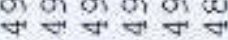

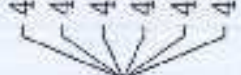

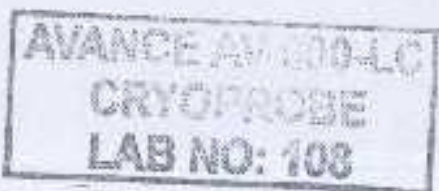

JXME
EXPAO

PROCNO

Date

Time-

PROBn:

PULPROG

77

SOLVSNT

หร

DE

sutares

AQ

$\mathrm{RG}$

DE

TE

CNST

52.

012
700

\section{NuC:}

koct

P1

:10

241

EI.19

SFO1

SE2

SENALI2

SEOAL2

FOTFS?

CFDPRE2

$\mathrm{suC} 2$

P3
P4
FCID2
FT.

Fito2

$\mathrm{LL12}$

$=12 \mathrm{~N}$

L 12 in

$\mathrm{ST}$

NON

LH

dec $23-13$

20131224 1. 5 $5 \mathrm{~mm}$ cPTC 5 pest deptapsc 32768 533
2 $30303.031 \mathrm{E2}$ $0.926775 \mathrm{~Hz}$ 32768 16.900 tasec 6.50 usec 145.0000000 145.0000000 $2+5000000$ sec $0.0034432 \mathrm{~d}$ sec .0002000 Chanvel f

$$
\begin{array}{r}
136 \\
12.70 \text { usec } \\
2000.46 \mathrm{usec} \\
120.00 \mathrm{dH} \\
-1.35 \mathrm{dH} \\
9.00900000 \mathrm{w} \\
81.92915344 \mathrm{w} \\
150.8927518 \mathrm{kHz} \\
4.19 \mathrm{~dB} \\
\text { Crpeccomp. } \\
0.500
\end{array}
$$
CHAMKE- Fy =waltz16 8.00 usec 16.00 usec 80.60 usec
3.31 di 23.31 dB 6. 79873323 o 0.06798734 w $600.0330002 \mathrm{MH2}$ 16354
4.004 $150.8774509 \mathrm{KHH}$ EM 1. $00=z$ 1.40 
NAILA RAZIQ/DR. SHAIQ/NR-03

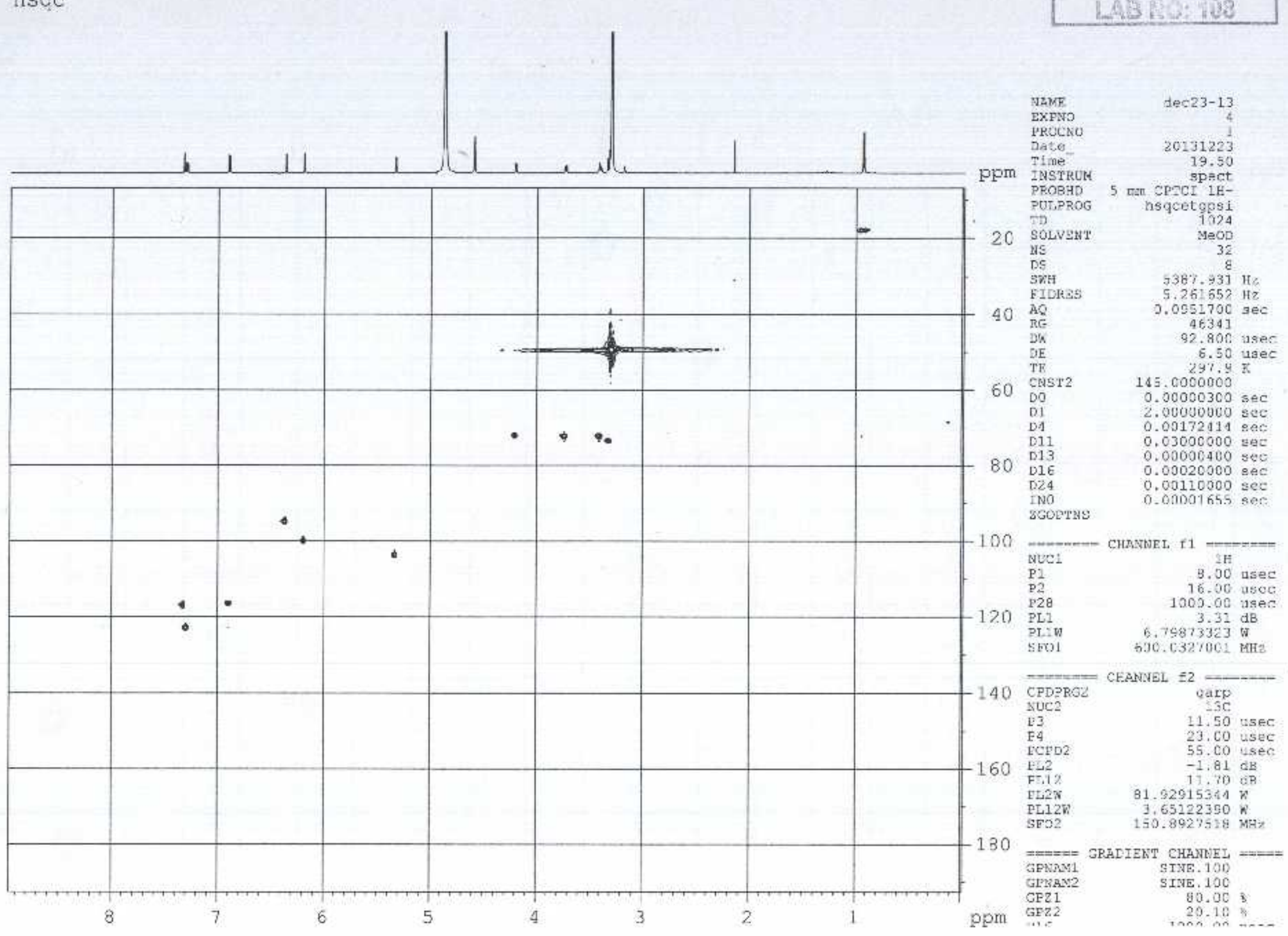


NAILA RAZIQ/DR.SHAIQ/NR-C 3

hmbe
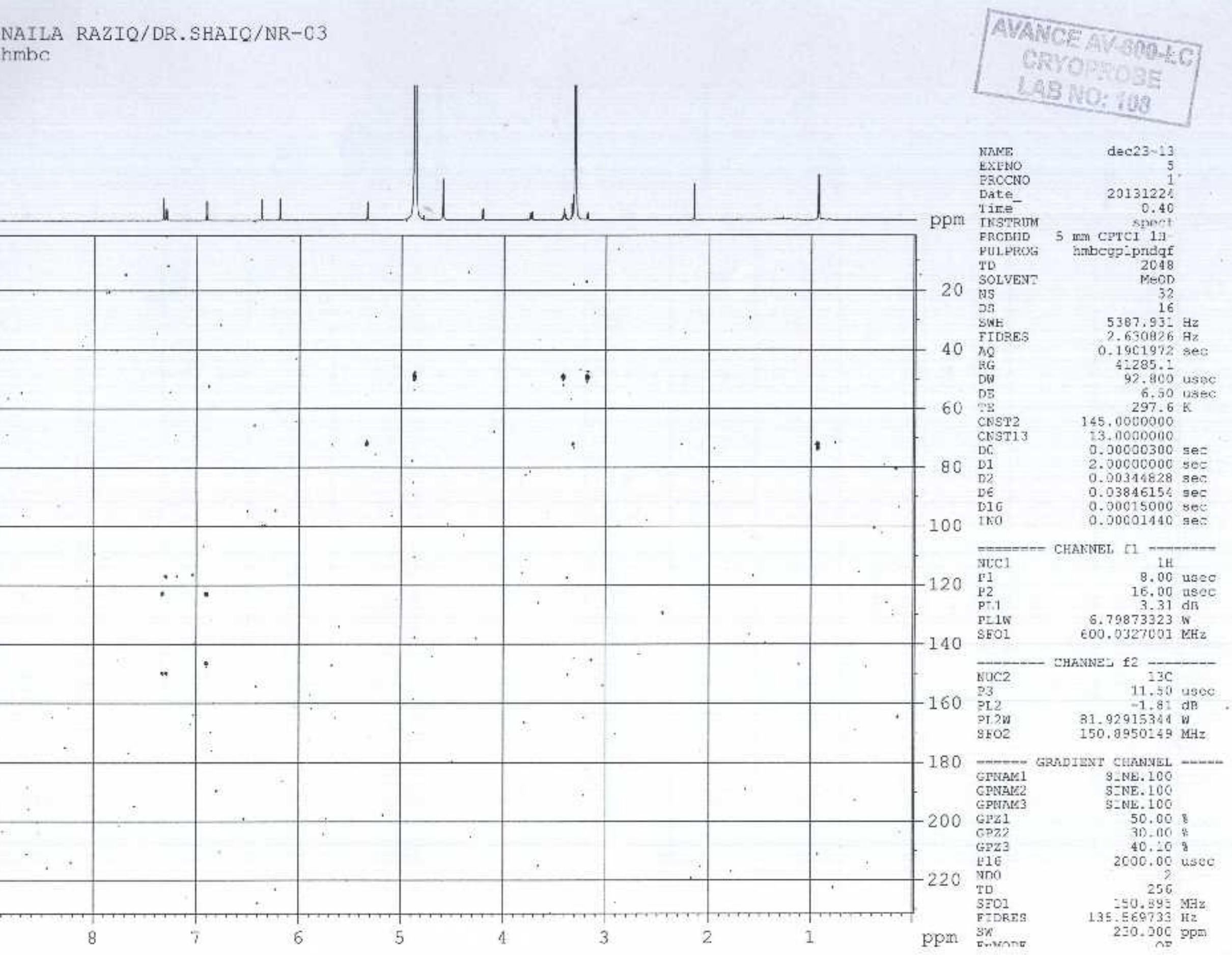


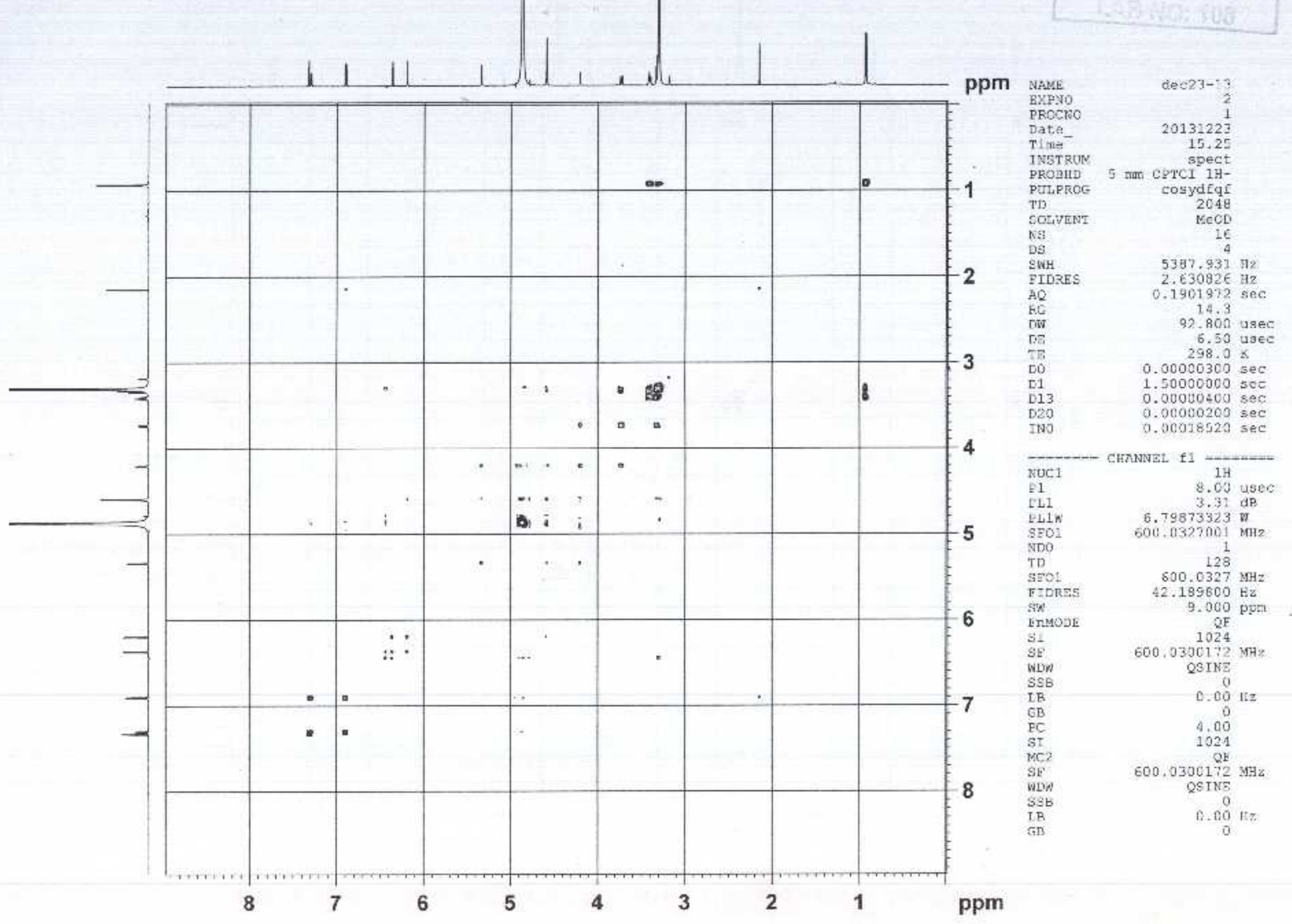




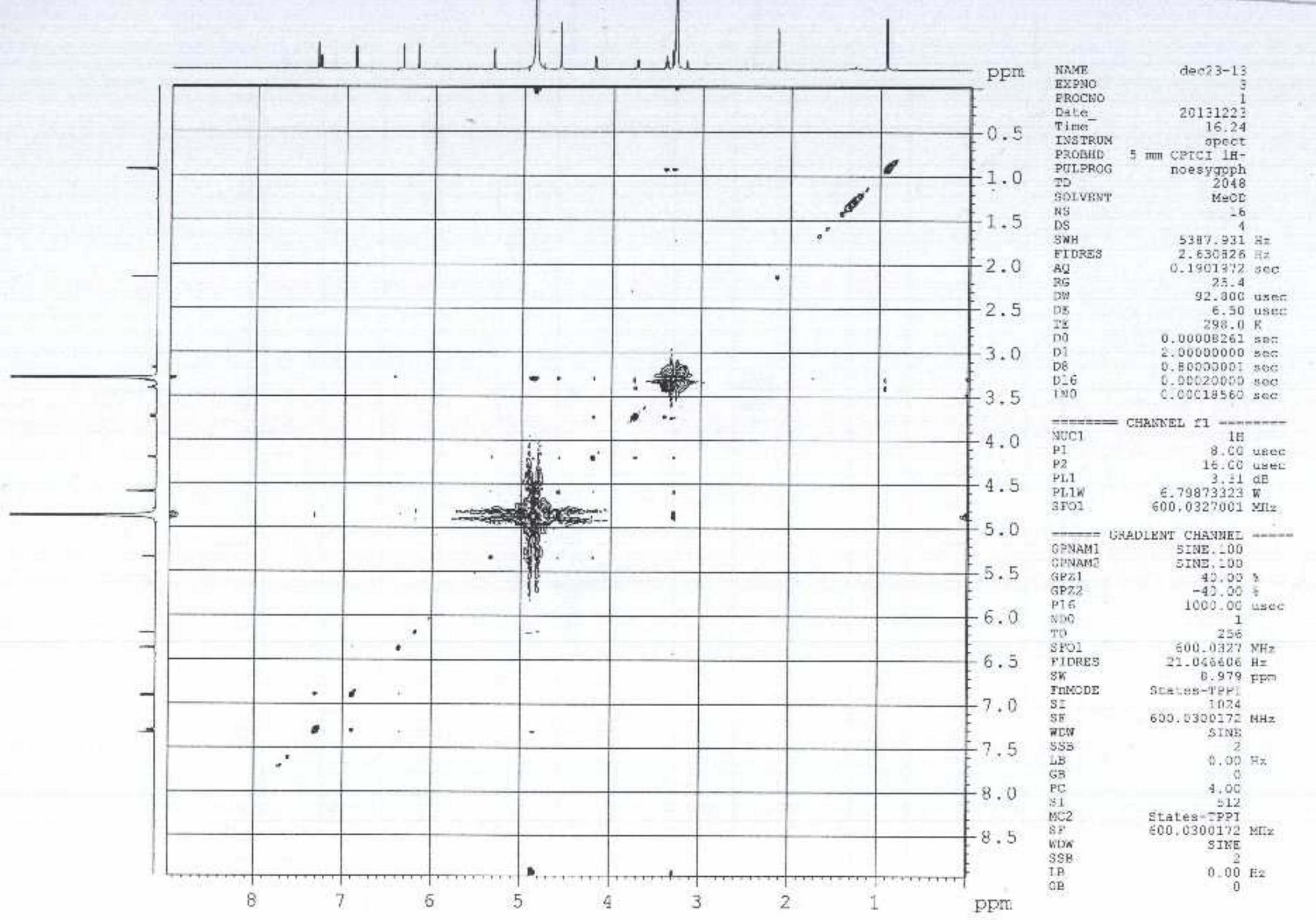


File: NR-03

Sample: NAILA RAZIAJDR, SHAIQ

Instrument: JEOL JMS 600-H

Inlet: My Intet

Date Run: 12-05-2013 (T ime Run: 15:47:28)

Ionization mode: $\mathbf{E H}+$

Scan: 24

Base: m/z 302; $99.9 \%$ FS TIC: 4289376

$100-$

$80-$

Galt

$-$

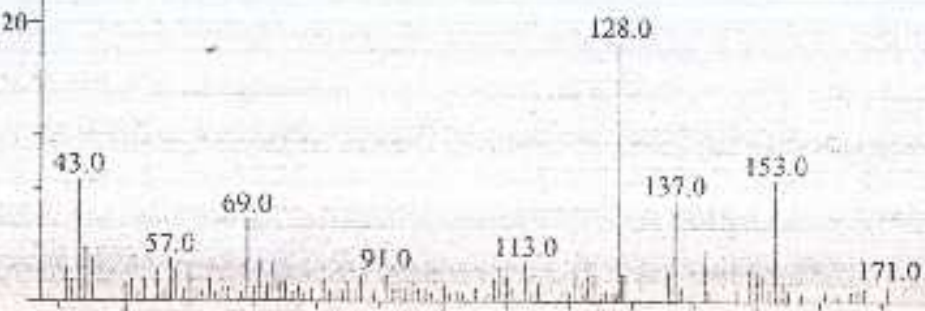

Sit $=100$
R.T.: 2.05

Hons: 136

3020

2740 
의 Г以

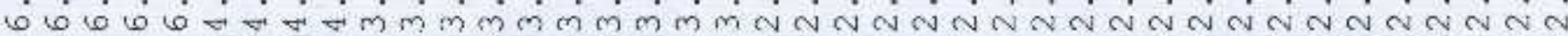
$+\infty$

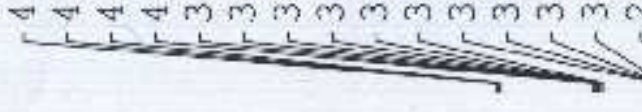

\section{NR $-01=$ Compound 2}

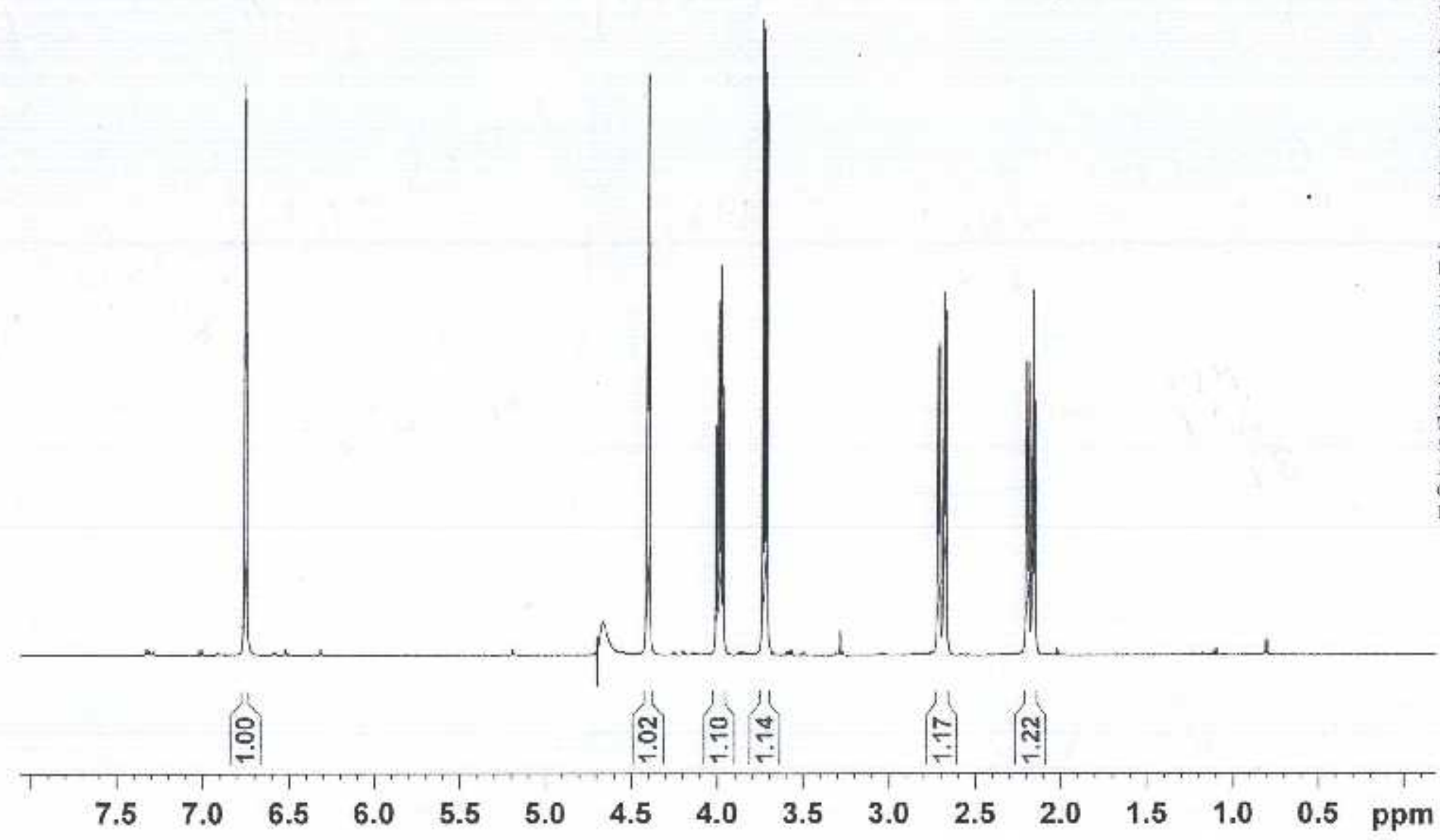

WRME EXPNO PROCNO Date

INSTRUY

PROEHD

PUI,PROG

TD

SOLVENT

NS

as

EIDRES

FIDI

$\mathrm{RG}$

DE

12

212

TDO

NuC.

PI. 7

PLS

SI

$S F$

GE

PC dec $20-13$

20131220 15.04 IM PABBI IH/ 29px
65536 D20 16

$5000.000 \mathrm{~Hz}$ 2. $076294 \mathrm{~Hz}$ 6.553/500 $\mathrm{sec}$ 574.7

110. 000 usec 6.50 usec $300.0 \mathrm{~K}$ 2.00scusoo sec $0.00002 \mathrm{co0} \mathrm{sec}$ ChARNALL Ё

$1 \mathrm{H}$
$8.13 \mathrm{usec}$
$5.00 \mathrm{AR}$
$59.80 \mathrm{~dB}$
$500.2323493 \mathrm{MHz}$
$32768 \mathrm{~Hz}$
$500.2299980 \mathrm{AFz}$
$\mathrm{MM}$
0
$0.30 \mathrm{~Hz}$
0
1.00




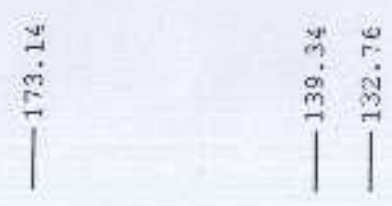

AVANCE $A N-500$

ITB NO: $109 \mathrm{~B}$

$\underset{\sim \infty}{\infty}$

mai

1 1)

in

1

\begin{tabular}{|c|c|c|c|c|}
\hline $\mathrm{ME}$ & \multicolumn{4}{|c|}{ jan08-14 } \\
\hline ExpHo & & & 21 & \\
\hline FROCNO & & & 1 & \\
\hline Date & & 201 & 40108 & \\
\hline Tine & & & 16.17 & \\
\hline INSTRUM & & & spect & \\
\hline EROBHD & 5 n⿺ & E3I & 1H/D- & \\
\hline $\begin{array}{l}\text { PULPROG } \\
T D\end{array}$ & & & zgpg & \\
\hline & & & $\begin{array}{r}32763 \\
720\end{array}$ & \\
\hline $\begin{array}{l}\text { SOLVENT } \\
\text { NS }\end{array}$ & & & $\begin{array}{r}020 \\
16384\end{array}$ & \\
\hline DS & & & 4 & \\
\hline SWH & & 2949 & 88.525 & $\mathrm{HZ}$ \\
\hline E'DEES & & 0.9 & 000224 & $\mathrm{~Hz}$ \\
\hline $\mathrm{AQ}$ & & 0.55 & $548 \$ 5$ & \\
\hline $\mathrm{RG}$ & & & 32768 & \\
\hline W & & & 6.950 & user \\
\hline $\mathrm{DE}$. & & & 6.50 & use \\
\hline ZE & & & 300,0 & \\
\hline D1 & & .900 & 100000 & sec \\
\hline D11 & & 0.030 & 100000 & sec \\
\hline TDO & & & 16 & \\
\hline & CLLARA & AEL $f$ & 21 & $=$ \\
\hline $\begin{array}{l}\text { nucl } \\
\mu 1\end{array}$ & & & & \\
\hline PLI & & & 00 & $d D$ \\
\hline \$EOI & & 85.77 & 23529 & $\mathrm{MHz}$ \\
\hline
\end{tabular}

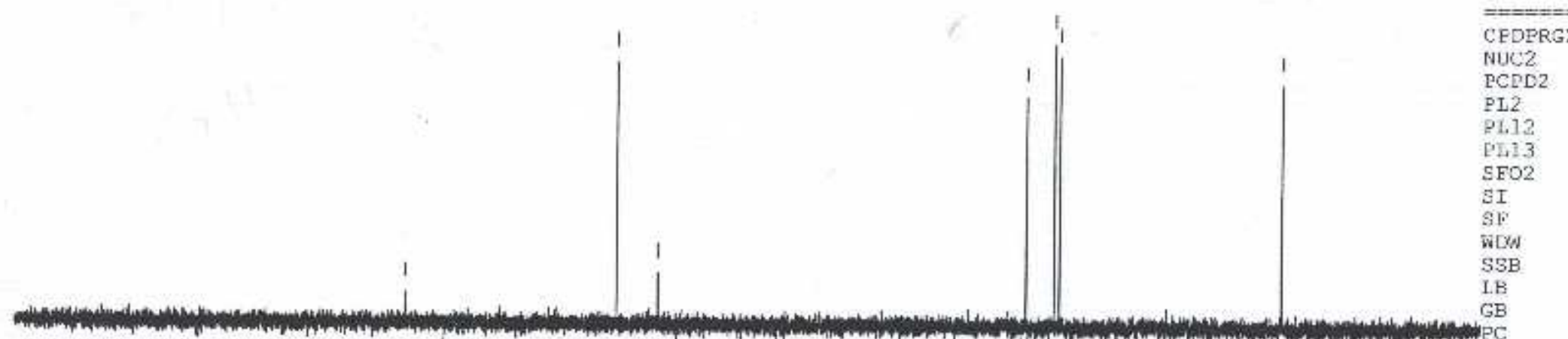

$125.7723529 \mathrm{MHz}$

$==$ CHANNEL

EDERG:

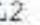

13

SSB

I.B

CHANNEL $E 2=$

$1 t=16$

30.00 usec

$-1.00 \mathrm{~dB}$

13.20 d

$22.00 \mathrm{~dB}$

500.1325007 MItz

125.7574612 MH $E M$
$C$

2. $00 \mathrm{~Hz}$

1.00

\begin{tabular}{rrrrrrrrrrrr}
\hline 220 & 200 & 180 & 160 & 140 & 120 & 100 & 80 & 60 & 40 & 20 & ppm
\end{tabular}


NAILA RAZIA/DR. SHAIQ ALI/NR-01/D2O DEPT 90

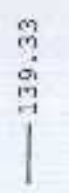

AVANCE AV-500

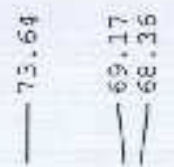

\begin{tabular}{|c|c|c|}
\hline WAME: & $\tan 08-14$ & \\
\hline EXpNo & 23 & \\
\hline PROCNO & 1 & \\
\hline Date_ & 20140109 & \\
\hline 'ime & 9.25 & \\
\hline ZNSTRUM & spect & \\
\hline PROAUD & $5 \mathrm{~mm} \mathrm{BBI} 1 \mathrm{H} / \mathrm{D}$ & \\
\hline $\begin{array}{l}\text { PULFROG } \\
\text { TD }\end{array}$ & dept spso & \\
\hline $\begin{array}{l}\text { TD } \\
\text { SOLVENT }\end{array}$ & $\begin{array}{r}32768 \\
020\end{array}$ & \\
\hline HS & 5120 & \\
\hline DS. & 2 & \\
\hline SWE & 23809.523 & $\mathrm{H}=$ \\
\hline FIDRES & $\begin{array}{r}0.726609 \\
0.6881990\end{array}$ & 112 \\
\hline & $\begin{array}{r}0.6881990 \\
32763\end{array}$ & $\sec$ \\
\hline DW & 21.000 & usec \\
\hline & 6.50 & usec \\
\hline & 300.0 & k \\
\hline CNST2 & 145.0000000 & \\
\hline D1 & 1.50000000 & $s \in C$ \\
\hline D2 2 & 0.00344828 & $\sec$ \\
\hline D12 & 0.00002000 & sec \\
\hline Тг. & 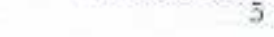 & \\
\hline NUC1 & IIANNEI II $=$ & \\
\hline P1. & 12.00 & uece \\
\hline P12. & 2000.20 & usec \\
\hline PLO & 120,00 & $d B$ \\
\hline PL1 & -3.00 & dî́ \\
\hline SFO1 & 125.7697360 & $\mathrm{KAHZ}$ \\
\hline $\mathrm{SP} 2$ & 3.58 & $d \mathrm{e}$ \\
\hline SFNRM 2 & Crp6ucomp.4 & \\
\hline $\begin{array}{l}\text { SPOAL2 } \\
\text { SPOEES2 }\end{array}$ & $\begin{array}{r}0.500 \\
0.00\end{array}$ & \\
\hline Shuet 32 & 0.00 & \\
\hline
\end{tabular}

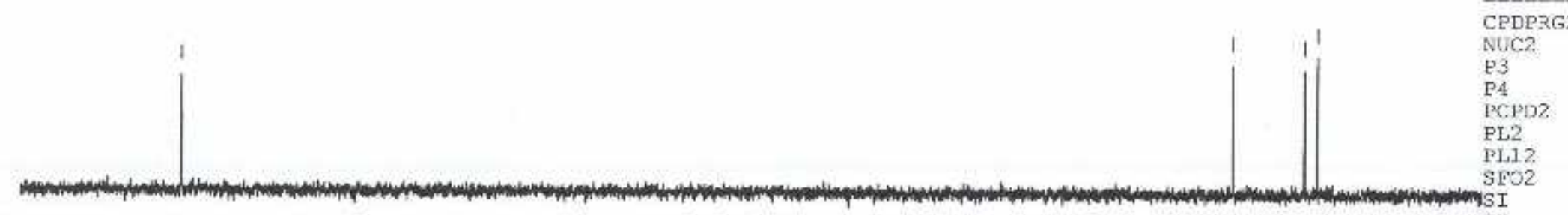

Hom

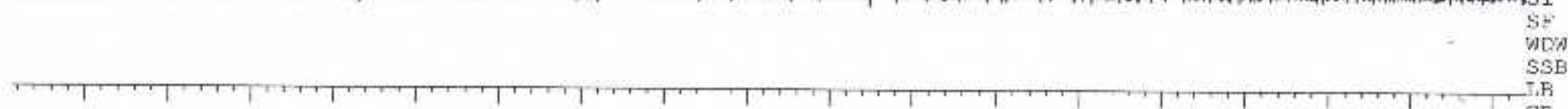

- CHRNNEL $12======$

DPRG

xCPD?

;

SI

waltz16
$1 \mathrm{H}$
8.00 usec
$16.00 \mathrm{uscc}$
$80.00 \mathrm{usec}$
$-1.00 \mathrm{iB}$
$19.20 \mathrm{~dB}$
$500.1335009 \mathrm{MHz}$
32768
$125.7574612 \mathrm{NHz}$
$\mathrm{EM}$
0
$1.00 \mathrm{~Hz}$
0
1.40

$\begin{array}{lllllllllllllllllllllll}145 & 140 & 135 & 130 & 125 & 120 & 115 & 110 & 105 & 100 & 95 & 90 & 85 & 80 & 75 & 70 & 65 & \mathrm{ppm} P \mathrm{C}\end{array}$ 
NAILA RAZIA/DR. SHAIQ ALT/NR-01/D20

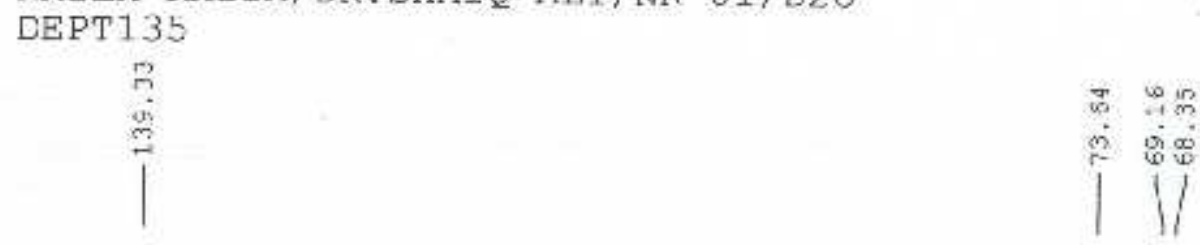

AVANCE RV-500

LAE NO: $109 \mathrm{~B}$

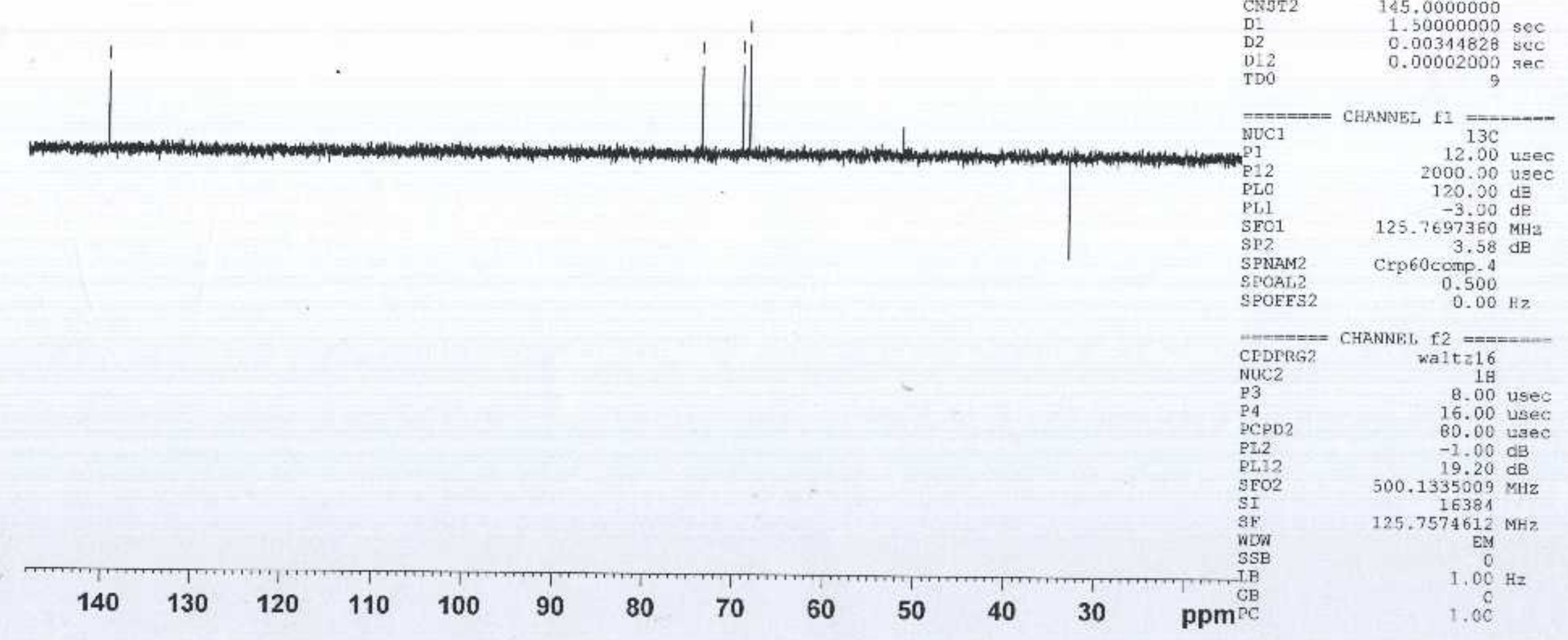




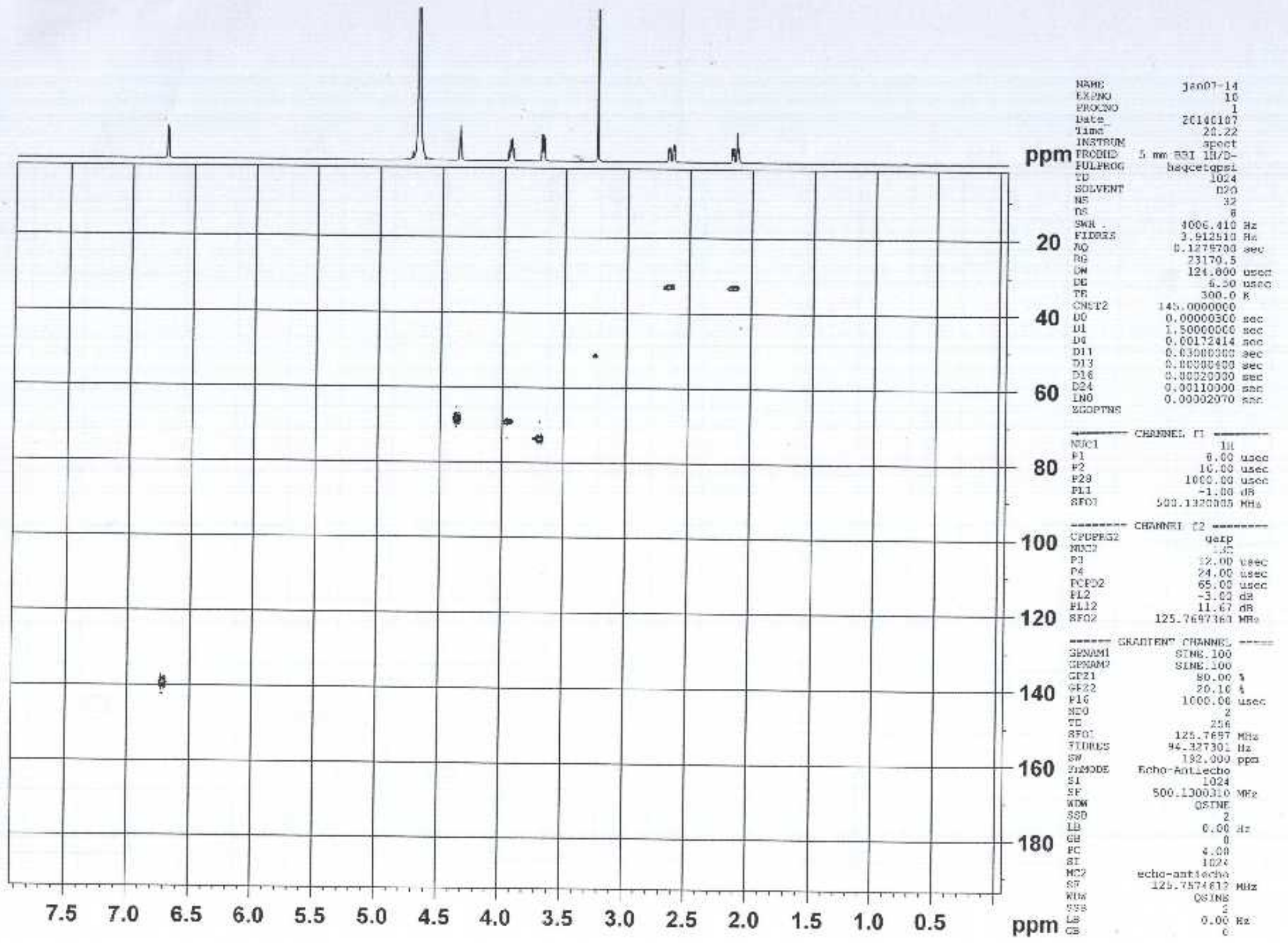




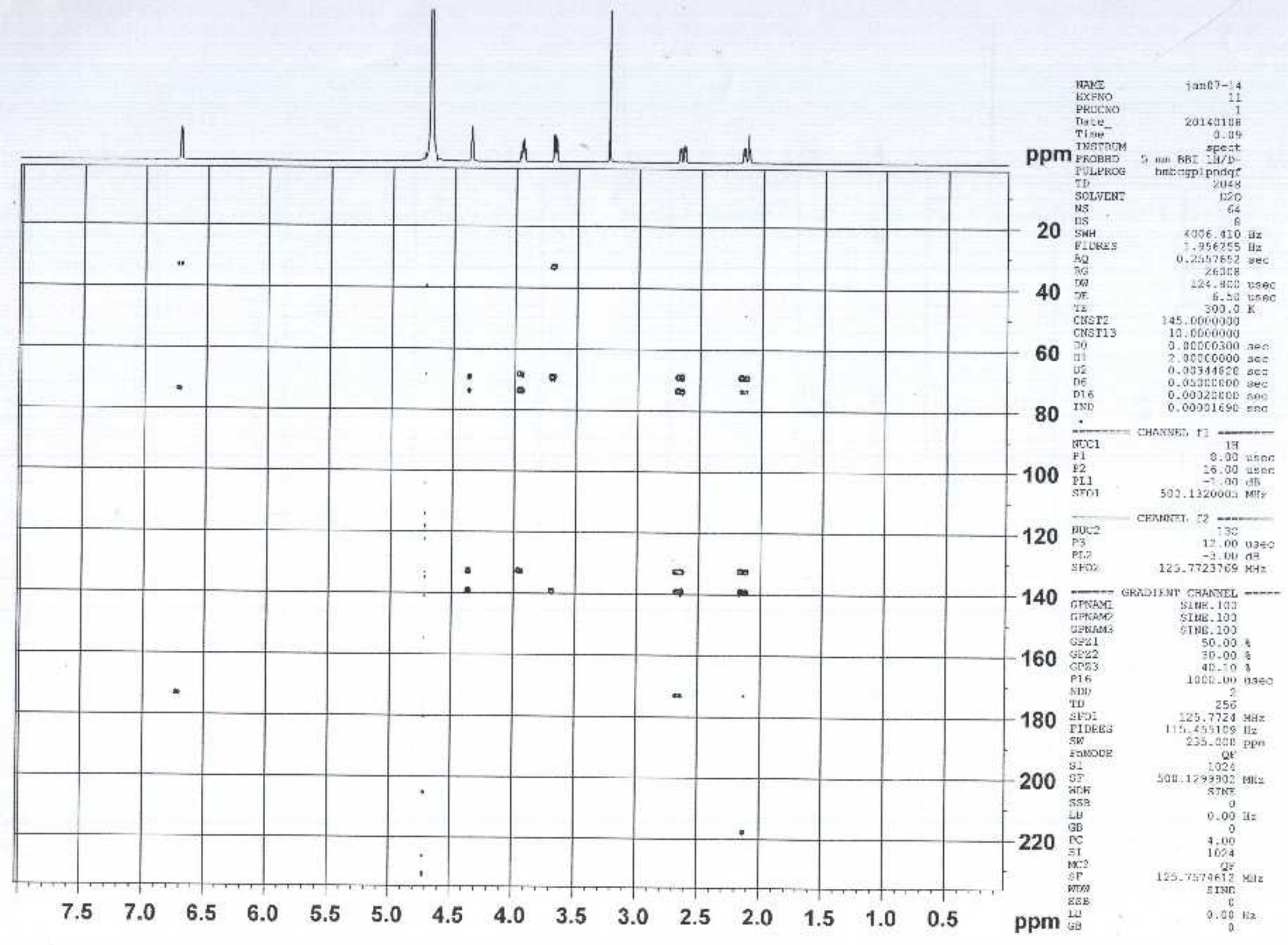


NAILA RAZIA/DR, SHAIO ALI/NR-01/D2O COSY
AVANCE:AV-500
XAB NO: $109 \mathrm{~B}$

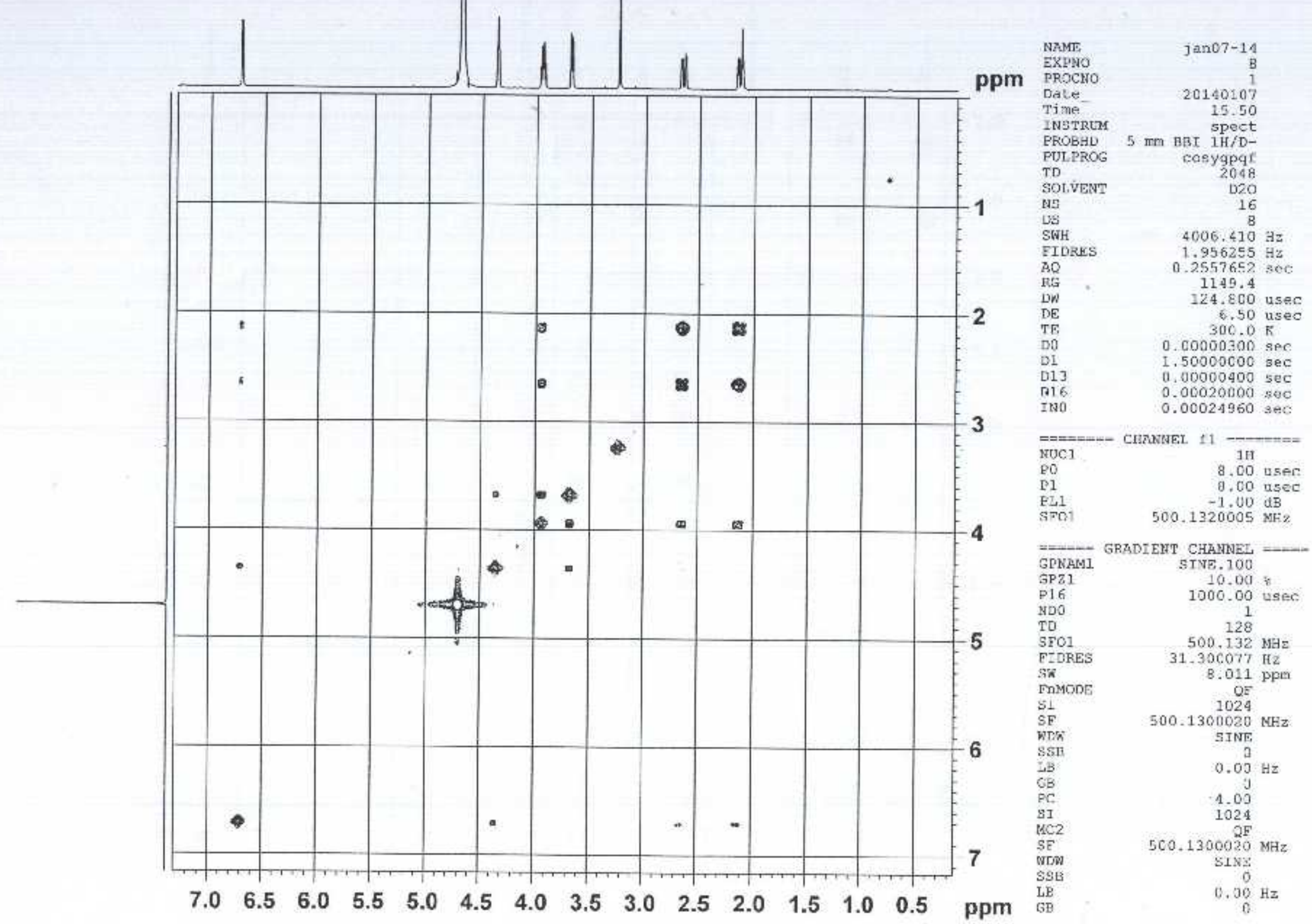




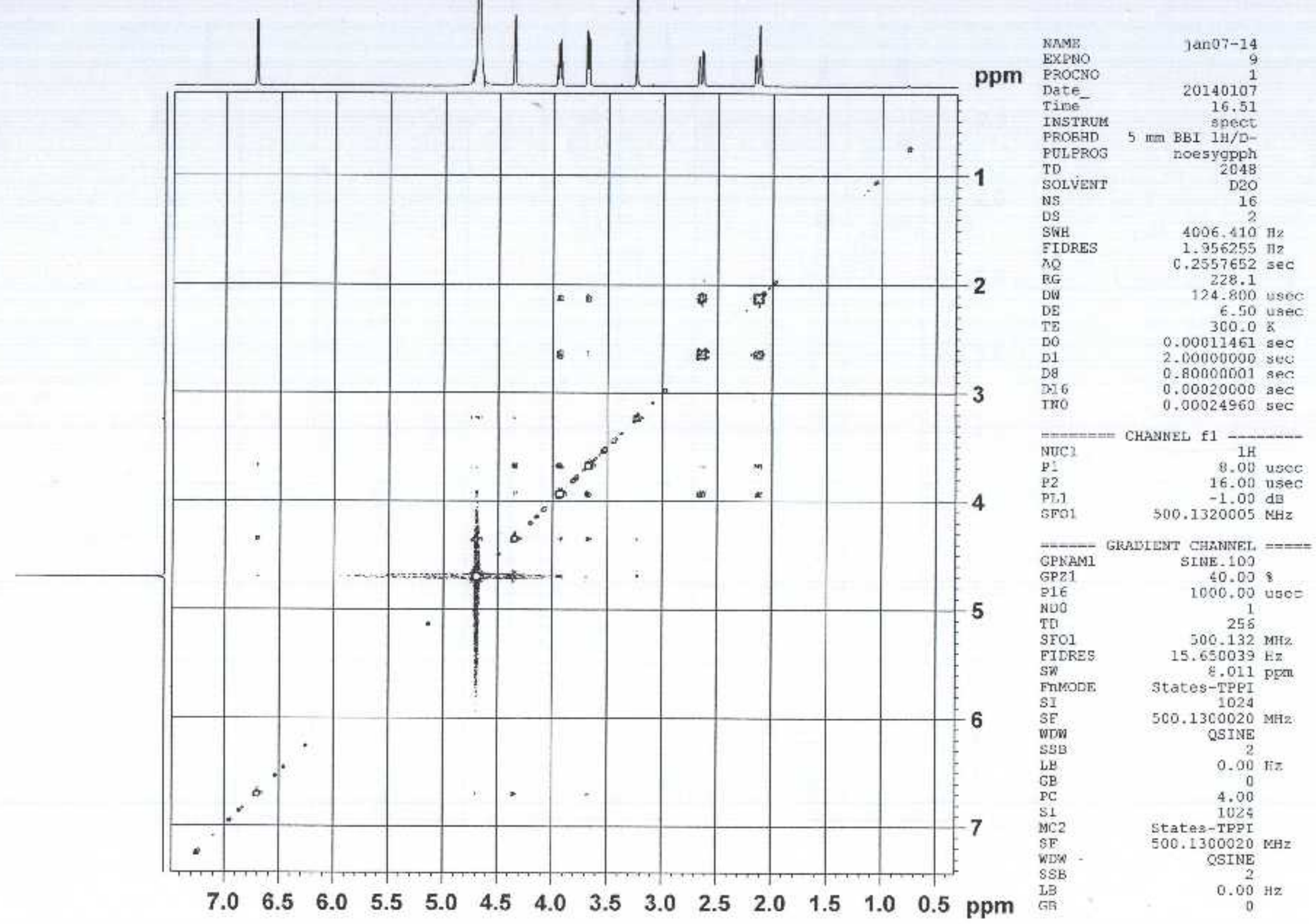


File: NR-01

Sample: NALLA RAZLAIDR. SHAIR ALT

Instrument: JEOL JMS 600-H

Inlet: My Intet

\section{Scan: 19}

Base: $\mathrm{m} / \mathrm{z}$ 97; $99.9 \% \mathrm{FS}$ TIC: 8668388

\section{Date Run: 12-19-2013 (Time Run: 14:57:15)}

\section{Ionization mode: EIt.}

\section{R.T: 1.6}

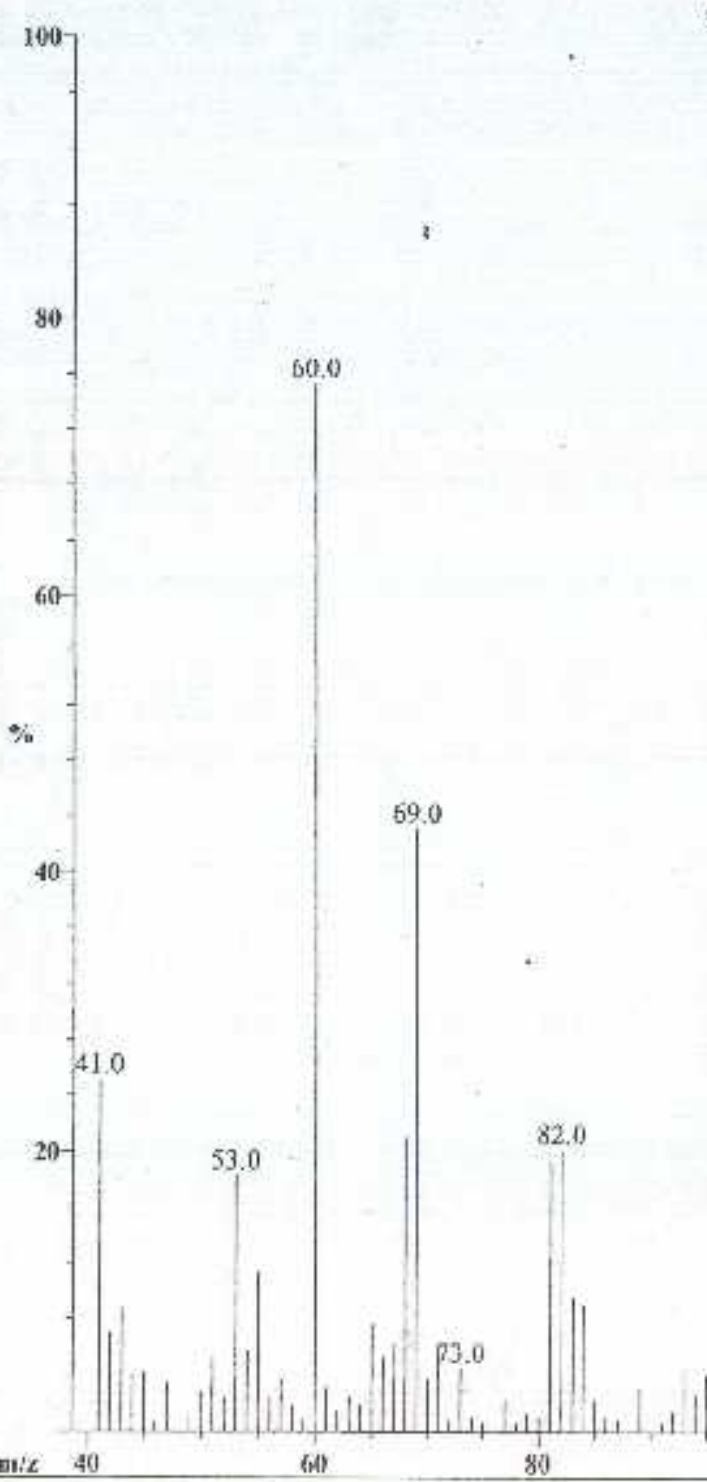

96.9

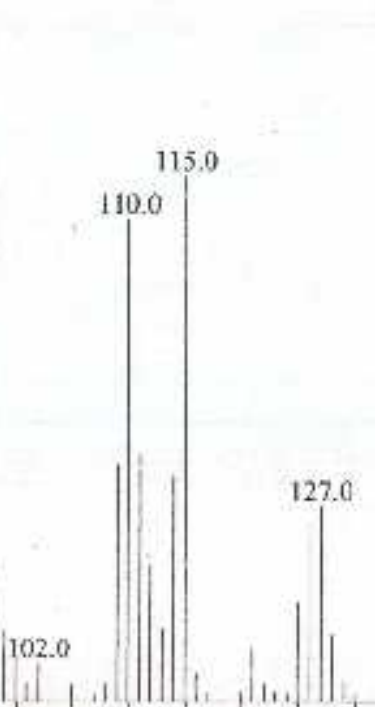

120 